\title{
Upfront Matched Unrelated Donor Transplantation in Aplastic
}

\section{Anemia}

\section{Authors}

Katherine Clesham ${ }^{1,2}{ }^{*}$, Robin Dowse ${ }^{1}$, Sujith Samarasinghe ${ }^{1}$

Affiliations

${ }^{1}$ UCL Great Ormond Street Hospital for Children, London, United Kingdom

${ }^{2}$ UCL GOS Institute of Child Health, London, United Kingdom

*- denotes equal contribution

Corresponding author:

Dr Sujith Samarasinghe

Department of Pediatric Hematology, UCL Great Ormond Street Hospital for Children, London, United Kingdom

Sujith.Samarasinghe@gosh.nhs.uk

\section{Key Words (5-8)}

Aplastic Anemia, Hemopoietic stem cell transplantation (HSCT), matched sibling donor, matched unrelated donor. 


\section{Abstract/Summary}

This article summaries the recent development in the field of front line unrelated donor transplantation for idiopathic aplastic anemia. The role of unrelated donor transplantation in the algorithm of pediatric aplastic anemia is reviewed and incorporates upfront unrelated donor transplantation. Newer strategies to delineate which children should receive immune suppression or transplantation are also discussed.

\section{Key Points}

- Idiopathic severe aplastic anemia (SAA) is a rare condition and haemopoietic stem cell transplantation (HSCT) remains the only curative therapy.

- HSCT is considered first line treatment in patients $<35$ with SAA with a matched sibling donor (MSD).

- Current best practice is less clear in those without a MSD. Immunosuppressive therapy (IST) has traditionally been thought of as first-line treatment in those without a MSD; however there remains a significant risk of relapse and clonal evolution.

- Outcomes following matched unrelated donor (MUD) HSCT in SAA have improved due to advances in the development of high resolution Human Leucocyte Antigen (HLA) typing, developments in supportive care and implementation of novel conditioning regimens. 
- After careful discussion with the patient and parents, MUD HSCT could be considered as first-line treatment in selected patients.

\section{Introduction}

Aplastic anemia (AA), defined by pancytopenia and a hypocellular marrow in the absence of reticulin fibrosis or abnormal infiltration. This rare disorder has an incidence of approximately 2 per million in Europe ${ }^{1}$. The majority of cases are idiopathic, with T-cell mediated destruction of haematopoietic stem cells thought to be the underlying pathophysiology. Without treatment patients are likely to succumb to infection or haemorrhage. In this review we will discuss how to approach one of the outstanding questions in the management of patients with SAA: 'What is the role of upfront MUD HSCT in aplastic anemia?'

\section{Treatment of Aplastic Anemia}

Allogeneic haematopoietic stem cell transplant (HSCT) from a human leucocyte antigen (HLA) matched sibling donor (MSD) is the preferred frontline treatment for pediatric and young adult patients with $A A^{1,2}$. In those lacking such a donor immunosuppressive therapy (IST) has historically been primary treatment, but with transplants from matched unrelated donors (MUD) improving in outcomes many centers now consider upfront MUD HSCT. Whilst historically MSD HSCT was associated with a higher overall survival than IST, this is no longer the case in children due to better salvage of IST failures. Recent studies from Japan and EBMT indicate that front line MSD HSCT lead to a better failure free 
survival than IST but not overall survival (OS) ${ }^{2-4}$. Therefore, due to the excellent OS seen in pediatric SAA, comparisons between IST and HSCT should focus on event free survival (EFS) rather than OS.

The use of IST as treatment for AA originates from the observation of autologous marrow reconstitution in patients with graft failure who had undergone HCST with ATG conditioning ${ }^{5}$. Current guidelines recommend horse Anti-Thymocyte Globulin (h-ATG) with cyclosporin (CSA), as combination therapy is superior to ATG alone ${ }^{6}$. Pediatric studies utilizing this combination have reported overall response rates of between 59.9 to $77 \%^{7-10}$. Factors influencing response rates are shown in Table 1.

The use of the more immunosuppressive rabbit ATG ( $r$-ATG) is not recommended outside of relapse/refractory cases as it has been associated with inferior outcomes compared with h-ATG ${ }^{8,11-13}$. Response to IST is evaluated between 3 and 4 months post treatment. If there has been a response, cyclosporin can be weaned slowly from 6 months with frequent monitoring of bloods for signs of relapse. Between $15-25 \%$ of children remain CSA dependent ${ }^{9}$. The monitoring required and ensuing subnormal blood counts may have a detrimental effect on children and their families' quality of life. Relapse rates have been reported in $11.9-33 \%$ of patients undergoing IST. Current practice is to use MUD HSCT for those failing one course of IST, due to superior EFS compared with second course of IST 14. In patients who have had a prior response to IST, in those without a suitable donor, a 
repeat IST has a response rate of $60-70 \%^{13,15}$. Between $10-15 \%$ of patients who have undergone IST for AA will go on to develop clonal evolution ${ }^{8,9}$ with the development of a significant Paroxysmal Nocturnal Haemoglobinuria (PNH) clone, myelodysplastic syndrome and/or acute myeloid leukaemia ( $A M L)$. This risk does not plateau, making of particular concern to the pediatric population. Advantages and disadvantages associated with IST and MUD HSCT are shown in Table 2 and 3. 


\section{Why MUD HSCT transplantation has improved}

Outcome after MUD HSCT for SAA has significantly improved over time due to a number of factors ${ }^{16}$. The availability of 'high resolution' (allelic) HLA molecular typing mean that donors who appear equally well matched on serological testing can be distinguished, thereby reducing rejection, graft versus host disease (GVHD) rates and thus improving overall survival. Development of less toxic conditioning regimens such as FCC (Fludarabine, cyclophosphamide and Campath (alemtuzumab)) have also significantly reduced rates of GVHD, whilst not increasing graft rejection, leading to improvements in overall survival ${ }^{17-19}$. A large EBMT study recently highlighted that OS for MUD HSCT is now approaching that of MSD HSCT, confirming that these developments were leading to tangible improvements ${ }^{20}$. We also recently published a report of 44 successive children who received a 10-antigen (HLA-A, -B, -C, -DRB1, -DQB1) MUD HSCT's, 40 of whom had previous IST. HSCT conditioning was a fludarabine, cyclophosphamide and alemtuzumab (FCC) regimen and did not include radiotherapy. There was an excellent estimated 5-year FFS of $95 \%$ with low rates of acute and chronic GVHD ${ }^{21}$. 


\section{Evidence of upfront MUD HSCT in SAA}

Following on from the excellent results that were seen in MUD HSCT post IST failure, we then determined the outcomes of front line MUD HSCT. Dufour et al, explored the feasibility and safety of upfront MUD transplantation in 29 children (aged between 0.518.6 years) from a UK cohort who received MUD HSCT (5 patients received mismatched unrelated donor (MMUD) HSCT) ${ }^{2}$. These patients lacked a MSD and did not receive prior IST. Outcomes measures were OS and EFS, which were derived from standard definitions used in previous AA publications ${ }^{22}$. Outcomes were compared with matched historical controls who had received:

(i) front-line MSD HSCT

(ii) front-line IST with horse ATG and cyclosporin and

(iii) MUD HSCT post IST failure as second-line therapy.

Time to neutrophil recovery in the MUD cohort was on average 18 days (range 9-29), translating to a median time from diagnosis to neutrophil recovery of 0.39 years. This compares favourably with average time to neutrophil recovery with IST of $0.25-0.33$ years.

Rates of GVHD were low with fludarabine, cyclophosphamide and alemtuzumab conditioning (FCC). The 1-year cumulative incidence (CI) of grade II-IV acute GVHD was 
$10 \% \pm 6 \%$, and $19 \% \pm 8 \%$ chronic GVHD (all cases were limited). One patient experienced graft failure after a 1-antigen mismatched transplant (this patient also had pre-existing HLA-A antibodies) and subsequently received a second successful HSCT. There was one death following MUD HSCT, due to idiopathic pneumonia syndrome after engraftment. The remaining 27 patients all achieved a complete remission.

There was no significant difference in OS and EFS between the upfront MUD/MMUD versus MSD controls ( $96 \%$ vs $91 \%, \mathrm{P}=0.3$ and $92 \%$ vs $87 \%, \mathrm{P}=0.37$, respectively) (Figure 1 ). Compared to IST controls, there was no significant difference between the OS seen in front line MUD HSCT cohort ( $94 \%$ vs $96 \%, \mathrm{P}=0.68$ ). There was however, significantly higher EFS in the MUD group vs IST control. (92\% vs 40\%, P=0.0001) (Figure 2). Lastly, comparison with MUD HSCT post IST failure controls revealed significantly higher OS and EFS (74\% vs $95 \%$ for both OS and EFS, $\mathrm{P}=0.02$ ) in the upfront MUD group (Figure 3 ). In the MUD post IST failure controls the median interval between diagnosis and HSCT was one year. An interval from diagnosis to HSCT greater than 6 months has been shown to adversely affects survival and this most likely explain the difference in OS seen between the two groups ${ }^{20}$. A short interval between diagnosis and HSCT is desirable as it reduces time to acquire infection or development of HLA antibodies from transfusions.

This study and others ${ }^{17}$ demonstrate that many of the factors that the previously made front line MUD HSCT undesirable i.e. severe GVHD, graft failure, treatment related 
mortality are no longer major concerns. Furthermore, the very high complete remission rates seen with MUD HSCT are particularly important in children to enable them to pursue a normal quality of life.

Both the UK studies are limited by being retrospective with the potential for selection bias. To counter this, a US prospective trial (TransIT, Trial number NCT02845596) is currently recruiting patients under 25 with SAA who lack a MSD to compare IST with horse ATG vs $10 / 10$ or $9 / 10$ MUD HSCT. This study will hopefully determine the safety and feasibility of front line MUD HSCT and lead to a comparison of EFS between IST and HSCT. 


\section{Factors affecting whether to undertake upfront MUD HSCT}

The decision to proceed with MUD or IST should be determined based on patient and donor factors (Table 4). Ethnic origin is a major determinant of a patient's likelihood of finding a matched unrelated donor. In one North American study, donors with a 7-8/8 HLA match were found in $90 \%$ of White/non-Hispanic patients, $76 \%$ of Hispanics, $62 \%$ of

Black/African Americans and $33 \%$ of Asians ${ }^{23}$. Thus for some patients from nonCaucasian backgrounds, front line MUD HSCT may not be a suitable option. Timing is highly relevant in whether to proceed with MUD. Patients should be tissue typed in the diagnostic work-up for SAA, to minimize delays in a MUD search if required. If a suitable unrelated donor cannot be found, or delays are anticipated with arranging the donation, then it is preferable to proceed with IST first-line, as delays in proceeding with IST could adversely affect response rates. 


\section{Counselling the patient and family}

The study by Dufour et $a l^{2}$, led the European blood and marrow transplant SAA Working Party (EMBT SAAWP) and the UK Children's Cancer and Leukemia Group (CCLG) to recommend that if a MUD can be found quickly, then HSCT may be considered an upfront treatment in children who lack a MSD. The UK paediatric idiopathic SAA algorithm is detailed in Figure 4. In the absence of prospective trials, then it is crucial to weigh up the factors for and against MUD HSCT in each case. It is recommended that all patients with SAA are cared for in centers with specialized experience in the management of children and adolescents with AA. A careful discussion with the patient and family to weigh up pros and cons of IST vs front line MUD HSCT is required. This can only be done in specialized hematology centres.

\section{Alternative Stem Cell Sources}

Haploidentical transplants are an attractive option due to rapid availability of a potential donor for most children. A retrospective multicentre data comparing haploidentical HSCT with matched sibling donor transplants showed similar overall survival (86.1 vs $91.3 \%$ ) and failure free survival ( 85 vs $89.8 \%)^{24}$. The haploidentical group did experience significantly higher rates of GVHD; nevertheless this data does suggest that in experienced centres, front line haploidentical HSCT is an emerging option especially when horse ATG or a MUD is readily not available 25,26 . A US prospective study (Trial number NCT02833805) 
is currently recruiting to determine the feasibility and safety of conducting front line MUD, partially matched and haploidential HSCT in SAA.

\section{Future directions of study}

Predicting which patients will respond to IST and who might develop clonal evolution may help to refine which patients may benefit from front line HSCT or IST. A study of 64 children with AA found that short telomere length (STL), as measured by flow cytometry, was a predictor of poor response to IST ${ }^{27}$. A follow up paper from the same group showed that the presence of a minor PNH clone was a predictive of good response to IST, and this was used to define good risk patients (PNH positive and/or longer telomere lengths)and poor risk (STL and no PNH clone). The good risk group showed a significantly better response to IST compared with poor risk ( $70 \%$ vs $19 \%, P<0.001){ }^{28}$. A Japanese study is planned to explore front line transplantation in children who have poor prognostic markers to IST i.e. STL and absence of PNH clone. Other biomarkers of IST response have been investigated. Very high levels of thrombopoietin at diagnosis is an independent predictor of poor response ${ }^{29}$, and the presence of intracellular interferon-gamma in patients lymphocytes may predict a good response to $\mathrm{IST}^{30}$.

With the advent of next generation sequencing (NGS) small populations of clonal haematopoiesis have been found in many patients with AA. Specific mutations may be predictive of poor response to IST and/or evolution to MDS/AML via immune escape and 
increased proliferation ${ }^{31}$. Analysis suggests that BCOR and BCORL1 mutations are associated with a good response to immunosuppressive therapy, while ASXL1, DNMT3A and RUNX1 mutations are linked with poor progression free and overall survival ${ }^{32}$. Those patients found to harbour these deleterious mutations may benefit most from transplant rather than IST, though this would need to be determined in future studies.

Improving outcomes in those undergoing IST appears to be possible with the addition of Eltrombopag, a thrombopoietin mimetic which has induced trilineage hemopoietic recovery in patients with IST-refractory $A A^{33}$. Recent studies have shown that the addition of Eltrombopag to IST leads to improved response rates in treatment naïve patients ${ }^{34}$. Further studies aim to recruit pediatric patients (NCT01623167) to assess efficacy and safety in this group. This development may lead to IST outperforming upfront MUD once again, although clonal evolution remains a potential concern, with some patients in these studies developing monosomy 7 and complex cytogenetic abnormalities ${ }^{35}$.

\section{Conclusions}

Upfront MUD HSCT is a safe and feasible treatment option for children and young adults with aplastic anemia when a suitable donor is readily available. Thus all patients who lack a MSD should have a donor search is initiated at diagnosis. The decision to proceed with transplant requires careful discussion with the patient and their family and should only be performed in a specialist centre experienced in the management of aplastic anemia. 


\section{Author contributions}

$\mathrm{RD}$ and $\mathrm{KC}$ wrote the draft manuscript. This was reviewed and edited by SS.

\section{Conflict of interests}

None to declare

\section{Acknowledgements}

Figures 1, 2 and 3 are taken from Dufour et al, Br J Haematol, 171: 585-594. Printed with kind permission of Wiley online library. 
References

1. Killick SB, Bown N, Cavenagh J, et al. Guidelines for the diagnosis and management of adult aplastic anaemia. British journal of haematology. 2016;172(2):187-207.

2. Dufour C, Veys P, Carraro E, et al. Similar outcome of upfront-unrelated and matched sibling stem cell transplantation in idiopathic paediatric aplastic anaemia. A study on behalf of the UK Paediatric BMT Working Party, Paediatric Diseases Working Party and Severe Aplastic Anaemia Working Party of EBMT. British journal of haematology. 2015;171(4):585-594.

3. Doney K, Leisenring W, Storb R, Appelbaum FR. Primary treatment of acquired aplastic anemia: Outcomes with bone marrow transplantation and immunosuppressive therapy. Annals of internal medicine. 1997;126(2):107-115.

4. Yoshida $\mathrm{N}$, Kobayashi $\mathrm{R}$, Yabe $\mathrm{H}$, et al. First-line treatment for severe aplastic anemia in children: bone marrow transplantation from a matched family donor versus immunosuppressive therapy. Haematologica. 2014;99(12):1784-1791.

5. Speck B, Gratwohl A, Nissen C, et al. Treatment of severe aplastic anaemia with antilymphocyte globulin or bone-marrow transplantation. British medical journal (Clinical research ed). 1981;282(6267):860-863.

6. Frickhofen $\mathrm{N}$, Heimpel H, Kaltwasser JP, Schrezenmeier H. Antithymocyte globulin with or without cyclosporin A: 11-year follow-up of a randomized trial comparing treatments of aplastic anemia. Blood. 2003;101(4):1236-1242.

7. Fuhrer M, Rampf U, Baumann I, et al. Immunosuppressive therapy for aplastic anemia in children: a more severe disease predicts better survival. Blood. 2005;106(6):2102-2104.

8. Scheinberg P, Nunez O, Wu C, Young NS. Treatment of severe aplastic anaemia with combined immunosuppression: anti-thymocyte globulin, ciclosporin and mycophenolate mofetil. British journal of haematology. 2006;133(6):606-611.

9. Saracco P, Quarello P, lori AP, et al. Cyclosporin A response and dependence in children with acquired aplastic anaemia: a multicentre retrospective study with long-term observation follow-up. British journal of haematology. 2008;140(2):197-205.

10. Kamio T, Ito E, Ohara A, et al. Relapse of aplastic anemia in children after immunosuppressive therapy: a report from the Japan Childhood Aplastic Anemia Study Group. Haematologica. 2011;96(6):814-819.

11. Yoshimi A, van den Heuvel-Eibrink MM, Baumann I, et al. Comparison of horse and rabbit antithymocyte globulin in immunosuppressive therapy for refractory cytopenia of childhood. Haematologica. 2014;99(4):656-663.

12. Takahashi Y, Muramatsu H, Sakata N, et al. Rabbit antithymocyte globulin and cyclosporine as first-line therapy for children with acquired aplastic anemia. Blood. 2013;121(5):862-863. 
13. Scheinberg $P$, Nunez $O$, Young NS. Retreatment with rabbit anti-thymocyte globulin and ciclosporin for patients with relapsed or refractory severe aplastic anaemia. British journal of haematology. 2006;133(6):622-627.

14. Kosaka $\mathrm{Y}$, Yagasaki $\mathrm{H}$, Sano $\mathrm{K}$, et al. Prospective multicenter trial comparing repeated immunosuppressive therapy with stem-cell transplantation from an alternative donor as second-line treatment for children with severe and very severe aplastic anemia. Blood. 2008;111(3):1054-1059.

15. Tichelli A, Passweg J, Nissen C, et al. Repeated treatment with horse antilymphocyte globulin for severe aplastic anaemia. British journal of haematology. 1998;100(2):393-400.

16. Locasciulli A, Oneto $R$, Bacigalupo A, et al. Outcome of patients with acquired aplastic anemia given first line bone marrow transplantation or immunosuppressive treatment in the last decade: a report from the European Group for Blood and Marrow Transplantation (EBMT). Haematologica. 2007;92(1):11-18.

17. Marsh JC, Gupta V, Lim Z, et al. Alemtuzumab with fludarabine and cyclophosphamide reduces chronic graft-versus-host disease after allogeneic stem cell transplantation for acquired aplastic anemia. Blood. 2011;118(8):23512357.

18. Marsh JC, Pearce RM, Koh MB, et al. Retrospective study of alemtuzumab vs ATGbased conditioning without irradiation for unrelated and matched sibling donor transplants in acquired severe aplastic anemia: a study from the British Society for Blood and Marrow Transplantation. Bone marrow transplantation. 2014;49(1):4248.

19. Samarasinghe S, lacobelli S, Knol C, et al. Impact of Different In Vivo T Cell Depletion Strategies on Outcomes Following Hematopoietic Stem Cell Transplantation for Idiopathic Aplastic Anaemia: A Study on Behalf of the EBMT SAA Working Party. Blood. 2015;126(23):1210-1210.

20. Bacigalupo A, Socie G, Hamladji RM, et al. Current outcome of HLA identical sibling versus unrelated donor transplants in severe aplastic anemia: an EBMT analysis. Haematologica. 2015;100(5):696-702.

21. Samarasinghe S, Steward C, Hiwarkar P, et al. Excellent outcome of matched unrelated donor transplantation in paediatric aplastic anaemia following failure with immunosuppressive therapy: a United Kingdom multicentre retrospective experience. British journal of haematology. 2012;157(3):339-346.

22. Dufour C, Pillon M, Passweg J, et al. Outcome of aplastic anemia in adolescence: a survey of the Severe Aplastic Anemia Working Party of the European Group for Blood and Marrow Transplantation. Haematologica. 2014;99(10):1574-1581.

23. Pidala J, Kim J, Schell M, et al. Race/ethnicity affects the probability of finding an HLA-A, -B, -C and -DRB1 allele-matched unrelated donor and likelihood of subsequent transplant utilization. Bone marrow transplantation. 2013;48(3):346350. 
24. Xu L-P, Jin S, Wang S-Q, et al. Upfront haploidentical transplant for acquired severe aplastic anemia: registry-based comparison with matched related transplant. Journal of Hematology \& Oncology. 2017;10(1):25.

25. Clay J, Kulasekararaj AG, Potter V, et al. Nonmyeloablative peripheral blood haploidentical stem cell transplantation for refractory severe aplastic anemia. Biology of blood and marrow transplantation : journal of the American Society for Blood and Marrow Transplantation. 2014;20(11):1711-1716.

26. Esteves I, Bonfim C, Pasquini R, et al. Haploidentical BMT and post-transplant Cy for severe aplastic anemia: a multicenter retrospective study. Bone marrow transplantation. 2015;50(5):685-689.

27. Sakaguchi H, Nishio N, Hama A, et al. Peripheral blood lymphocyte telomere length as a predictor of response to immunosuppressive therapy in childhood aplastic anemia. Haematologica. 2014;99(8):1312-1316.

28. Narita A, Muramatsu H, Sekiya $\mathrm{Y}$, et al. Paroxysmal nocturnal hemoglobinuria and telomere length predicts response to immunosuppressive therapy in pediatric aplastic anemia. Haematologica. 2015;100(12):1546-1552.

29. Elmahdi S, Muramatsu H, Narita A, et al. Markedly High Plasma Thrombopoietin (TPO) Level is a Predictor of Poor Response to Immunosuppressive Therapy in Children With Acquired Severe Aplastic Anemia. Pediatric blood \& cancer. 2016;63(4):659-664.

30. Sloand E, Kim S, Maciejewski JP, Tisdale J, Follmann D, Young NS. Intracellular interferon- $\gamma$ in circulating and marrow T cells detected by flow cytometry and the response to immunosuppressive therapy in patients with aplastic anemia. Blood. 2002;100(4):1185-1191.

31. Babushok DV, Perdigones N, Perin JC, et al. Emergence of Clonal Hematopoiesis in the Majority of Patients with Acquired Aplastic Anemia. Cancer genetics. 2015;208(4):115-128.

32. Yoshizato T, Dumitriu B, Hosokawa K, et al. Somatic Mutations and Clonal Hematopoiesis in Aplastic Anemia. New England Journal of Medicine. 2015;373(1):35-47.

33. Olnes MJ, Scheinberg $P$, Calvo KR, et al. Eltrombopag and Improved Hematopoiesis in Refractory Aplastic Anemia. New England Journal of Medicine. 2012;367(1):11-19.

34. Townsley DM, Scheinberg P, Winkler T, et al. Eltrombopag Added to Standard Immunosuppression for Aplastic Anemia. The New England journal of medicine. 2017;376(16):1540-1550.

35. Desmond R, Townsley DM, Dumitriu B, et al. Eltrombopag restores trilineage hematopoiesis in refractory severe aplastic anemia that can be sustained on discontinuation of drug. Blood. 2014;123(12):1818-1825. 